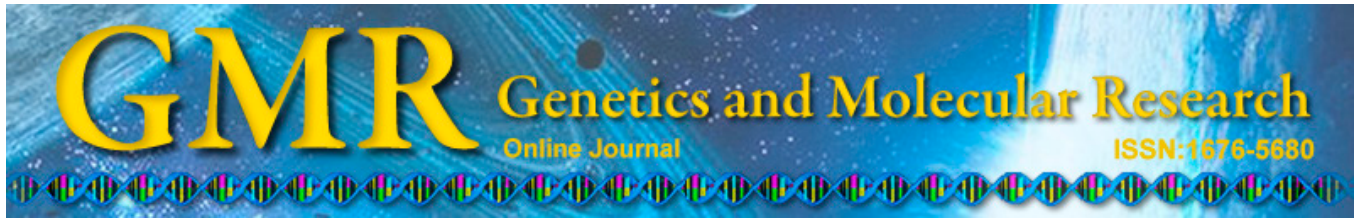

\title{
A clinical study evaluating dendritic and cytokine-induced killer cells combined with concurrent radiochemotherapy for stage IIIB non-small cell lung cancer
}

\author{
X.P. Zhu ${ }^{1,4 *}$ Y.H. Xu' ${ }^{2}$, J. Zhou ${ }^{3 *}$ and X.F. Pan $^{3}$ \\ ${ }^{1}$ Jingzhou Clinical Medical College, Yangtze University Jingzhou, Jingzhou, \\ Hubei Province, China \\ ${ }^{2}$ Jingzhou Hospital Affiliated to Tongji Medical College of Huazhong \\ University of Science and Technology Jingzhou, Jingzhou, Hubei Province, China \\ ${ }^{3}$ Jingzhou Central Hospital, Jingzhou, Hubei Province, China \\ ${ }^{4}$ Internal Medicine-Oncology, \\ Puai Hospital Department of Huangshi Central Hospital, Jingzhou, Hubei \\ Province, China \\ *These authors contributed equally to this study. \\ Corresponding author: Y.H. Xu \\ E-mail: xyhua666@163.com
}

Genet. Mol. Res. 14 (3): 10228-10235 (2015)

Received September 15, 2014

Accepted January 19, 2015

Published August 28, 2015

DOI http://dx.doi.org/10.4238/2015.August.28.6

\begin{abstract}
To compare the efficacy of dendritic and cytokineinduced killer cells (DC-CIK) therapy combined with concurrent radiochemotherapy on stage IIIB non-small cell lung cancer. Sixtythree patients with stage IIIB non-small cell lung cancer were randomly divided into the study and control groups. The study group, comprising 30 patients, was treated with DC-CIK combined with docetaxel-cisplatin chemotherapy and synchronization conformal radiotherapy. The control group including 33 patients was only treated with docetaxel-cisplatin chemotherapy and synchronization conformal radiotherapy. The
\end{abstract}


efficacy, Karnofsky performance score (KPS), tumor markers, 6-month and 12-month survival rate, $T$ cell subsets, and adverse reactions of the two groups were compared. The response rate of the study group was $83.3 \%(25 / 30)$, and that of the control group was only $54.5 \%(18 / 33)$. Furthermore, the KPS, T cell subsets, and 12-month survival rate was significantly higher in the study group, and there were significant differences between the two groups. The two groups had no significant difference in adverse reactions. The combined DC-CIK therapy, with synchronous radiotherapy and chemotherapy to treat stage IIIB nonsmall cell lung cancer was superior to single synchronous radiotherapy and chemotherapy. The combined therapy can improve the life quality and prolong the survival time of the patients.

Key words: Dendritic cells; Cytokine-induced killer cells; Concurrent chemoradiotherapy; Non-small cell lung cancer

\section{INTRODUCTION}

The morbidity and mortality rates of lung cancer are the highest in malignant tumors, and show a growing trend (Casal Rubio et al., 2014). Non-small cell lung cancer accounts for about $75 \%$ of all lung cancer patients, and shows high morbidity, high mortality and low survival rates (Chang, 2011); moreover, most patients are diagnosed in the advanced stages (NSCLC Meta-Analyses Collaborative Group, 2008). The 5-year survival rate of lung cancer was from $4-15 \%$. Platinum-containing two-drug combination chemotherapy is the first-line chemotherapy strategy for non-small cell lung cancer (Zhi et al., 2013; Pereira et al., 2013). Currently, concurrent chemoradiotherapy is the standard treatment for stage IIIB non-small cell lung cancer (Takayama et al., 2013), with more superior efficacy than sequential treatment (Bearz et al., 2013). However, concurrent chemoradiotherapy can bring about severe adverse reactions and significant decline in immune function, resulting in decreased tolerance. In recent years, with the ongoing studies on the mechanism of tumorigenesis and tumor development, tumor immune therapy has become an important part of comprehensive cancer treatment. Particularly, dendritic cells (DC) and cytokine-induced killer cells (CIK) play an important role in tumor immune therapy, owing to their broad anti-tumor activity as well as a progressively enhanced anti-tumor effect (Yang et al., 2013). The efficacy of DC-CIK combined with concurrent radiochemotherapy for stage IIIB non-small cell lung cancer was reported in this study.

\section{MATERIAL AND METHODS}

\section{Subjects}

\section{Inclusion criteria}

We included patients with non-small cell lung cancer confirmed by histopathology that was not treated by chemotherapy; whose survival time was expected to be greater than three months; who were older than 18 years; who were generally fair; with Karnofsky perfor- 
mance score (KPS) higher than 70 points; with normal blood, liver and kidney functions; with no serious complications; with confirmed stage IIIB based on clinical manifestations, physical examination, computed tomography (CT), magnetic resonance imaging, and bone Emission Computed Tomography; and with lesions to be measured.

\section{Exclusion criteria}

We excluded patients with CNS metastases; active infection; blood, liver, and kidney dysfunction; or serious complications. Patients who were pregnant or breast-feeding and who were allergic to docetaxel and cisplatin were also excluded.

\section{Exit criteria}

Patients with serious adverse reactions, and those who could not tolerate the treatment were required to withdraw.

\section{General information}

According to the 7th American Joint Committee on Cancer Staging manual, stage IIIB was T1-4N3M0, T4N2M0. Sixty-five patients with stage IIIB non-small cell lung cancer were enrolled in the study from January 2012 to November 2012, including 35 male patients and 30 female patients, ranging from 50 to 75 years in age. Patients were informed of relevant rules, and informed consent was collected. After receiving approval from the Ethics Committee of the hospital, 65 patients were randomly divided into two groups. The study group (treated with DC-CIK combined with chemoradiotherapy) contained 30 patients (17 males and $13 \mathrm{fe}-$ males; median age, 64 years). Of these, there were 17 cases with squamous cell carcinoma, 11 cases with adenocarcinoma, and 2 cases with large cell carcinoma. The control group (treated with concurrent chemoradiotherapy) contained 35 patients: one patient quit owing to serious adverse reactions, and another patient could not tolerate the treatment and was required to withdraw. In all, 33 cases were evaluated (18 males and 15 females; median age, 63 years); of these, there were 18 cases with squamous cell carcinoma, 13 cases with adenocarcinoma, and 2 cases with large cell carcinoma.

\section{Preparation of DC and CIK}

Anticoagulated peripheral blood $(100 \mathrm{~mL})$ was collected from the patients. Mononuclear cells were isolated by Ficoll density gradient centrifugation, and washed with $0.9 \%$ saline thrice. The cells were incubated for $2 \mathrm{~h}$ at $37^{\circ} \mathrm{C}$, under $5 \% \mathrm{CO}_{2}, 95 \%$ relative humidity using RPMI-1640 culture medium. Then the cell suspension was isolated and seeded in another culture flask. Interleukin-4 (IL-4) and granulocyte-macrophage colony-stimulating factor were added to the culture flasks of adherent cells immediately; tumor necrosis factor-alpha (TNF- $\alpha$ ) was added on the 5th day. Interferon-gamma (IFN- $\gamma$ ) was added to the culture flasks of suspension cells immediately; IL-1, IL-2, and anti-CD3 mAb were added on the 2nd day. Culture medium was changed regularly. DC and CIK cells were co-cultured at a ratio of 1:10 on the 7th day using RPMI-1640 culture medium containing IL-2. On the 12th day, DC and CIK cells were collected, washed, sub-bagged, mixed with $5 \%$ human serum albumin, and in- 
travenously administered for two or three transfusions. Cell cultures were regularly monitored for cell viability, bacteria, fungi, endotoxin, Mycoplasma, and cell phenotypes during culturing. All indicators were required to meet the standards before reinfusion.

\section{Treatment}

The chemotherapy regimen included the intravenous infusion of docetaxel $[75 \mathrm{mg} /$ $\mathrm{m}^{2}$ on day 1 (D1)] and cisplatin ( $25 \mathrm{mg} / \mathrm{m}^{2}$ from D1-3), for a cycle of 21 days, for a total of four cycles as co-chemotherapy. For the radiotherapy regimen, all patients underwent threedimensional conformal radiotherapy while undergoing concurrent chemotherapy; $5 \mathrm{~mm} \mathrm{CT}$ was used to enhance the scan and simulator after fixing the membrane. Gross tumor volume (GTV) contained primary tumor and lymph node metastasis; GTV outwardly expanded from 6-8 $\mathrm{mm}$ to form the clinical target volume (CTV), while the CTV outwardly expanded by approximately $10-20 \mathrm{~mm}$ forming planning target volume. Special attention was paid to reduce the dose in order to avoid threatening the lung, spinal cord, and heart. Radiation treatment required dose volume histogram assessment; for the whole lung, volume receiving at least $20 \mathrm{~Gy}$ (V20) was no more than $30 \%$, for the spinal cord, the maximum dose was lower than $45 \mathrm{~Gy}$, and for the heart, V40 was lower than $40 \%$. Radiotherapy was prescribed at a dose of $200 \mathrm{cGy} /$ day, 5 times per week, at a tumor dose of approximately 60-70 Gy. For DC-CIK immune cell therapy, in the two days prior to chemotherapy, blood was collected and cells were cultured in vitro for 12 days, and subsequently administered two or three times through intravenous transfusion. The regimen was a 21-day cycle, for a total four cycles.

\section{Evaluation criteria}

According to the World Health Organization's (WHO's) response evaluation criteria in solid tumors (Tsuchida and Therasse, 2001), efficacy is divided into complete remission (CR), partial remission (PR), stable disease (SD), and progressive disease (PD). The response rate $(\mathrm{RR})=(\mathrm{CR}+\mathrm{PR}) /$ total $\times 100 \%$; and disease control rate $(\mathrm{DCR})=(\mathrm{CR}+\mathrm{PR}+\mathrm{SD}) /$ total $\mathrm{x} 100 \%$. T cell subsets were identified in order to conduct an immunological evaluation. Adverse reactions of chemotherapy (based on the toxicity criteria of anticancer drugs by the WHO) and radiotherapy (based on the radiation therapy oncology group/European organization for research and treatment of cancer standards) were divided into degrees I, II, III, and IV.

\section{Statistical analyses}

Statistical analyses were conducted using SPSS version 17.0. Chi-square tests were performed for count data, and the Student $t$-test was conducted to test measurement data; a P $\leq 0.05$ was considered to be significant.

\section{RESULTS}

\section{Comparison of the two groups' short-term response rates}

There was a significant difference $(\mathrm{P}=0.014)$ in the $\mathrm{RR}$, which was $83.3 \%$ in the study group, and $54.5 \%$ in the control group (Table 1). 


Table 1. Comparison of curative effect [N (\%)].
\begin{tabular}{lcccccrr}
\hline Group & N & CR & PR & SD & PD & RR & DCR \\
\hline Research group & 30 & $9(30.0)$ & $16(53.3)$ & $2(6.7)$ & $3(10.0)$ & $25(83.3)$ & $27(90.0)$ \\
Control group & 33 & $6(18.2)$ & $12(36.4)$ & $10(30.3)$ & $5(15.2)$ & $18(54.5)$ & $28(84.8)$ \\
\hline
\end{tabular}

\section{Comparison of the two groups' KPS}

Before treatment, the KPS in the study group was $75.0 \pm 6.3$ while it was $74.2 \pm 6.1$ in the control group $(\mathrm{P}>0.05)$. After treatment, the KPS in the study group was $78.7 \pm 10.4$, while it was $72.1 \pm 10.5$ in the control group $(\mathrm{P}<0.05$, Table 2$)$.

Table 2. KPS score compared with one week before treatment and four works after treatment.

\begin{tabular}{lcr}
\hline Group & & KPS score \\
\cline { 2 - 3 } & Before treatment & After treatment \\
\hline Research group & $75.0 \pm 6.3$ & $78.7 \pm 10.4$ \\
Control group & $74.2 \pm 6.1$ & $72.1 \pm 10.5$ \\
P & $>0.05$ & $<0.05$ \\
\hline
\end{tabular}

\section{Changes in the tumor markers after treatment}

Peripheral blood samples were obtained one week before treatment and four weeks after treatment. The level of carcinoembryonic antigen (CEA), cancer antigen 125 CA125 and cytokeratin 19 fragments (CYFRA211) were tested. After treatment, the levels of CEA, CA125, and CYFRA211 decreased significantly in both groups as compared to levels before treatment; with a statistical difference; however, there was no significant difference between the two groups (Table 3).

Table 3. Before and after treatment of tumor markers.
\begin{tabular}{lllllc}
\hline Group & $\mathrm{N}$ & & \multicolumn{3}{c}{ Tumor markers } \\
\cline { 3 - 6 } & & & CEA $(\mathrm{ng} / \mathrm{mL})$ & CA125 $(\mathrm{U} / \mathrm{mL})$ & CYFRA211 (ng/mL) \\
\hline Research group & One week before treatment & 30 & $27.14 \pm 6.38$ & $78.72 \pm 16.97$ & $13.05 \pm 4.23$ \\
& 4 weeks after treatment & 30 & $13.52 \pm 4.42^{\Delta}$ & $37.12 \pm 11.22^{\Delta}$ & $6.29 \pm 3.17^{\Delta}$ \\
Control group & One week before treatment & 33 & $27.43 \pm 6.53$ & $76.79 \pm 17.69$ & $12.82 \pm 5.05$ \\
& 4 weeks after treatment & 33 & $14.42 \pm 5.70^{\Delta}$ & $41.75 \pm 14.17^{\Delta}$ & $6.65 \pm 3.25^{\Delta}$ \\
\hline
\end{tabular}

One week before treatment, ${ }^{\Delta} \mathrm{P}<0.05$.

\section{Survival rate follow-up}

The patients' detailed information, including addresses and telephone numbers, were recorded when admitted to hospital; 63 patients were followed up regularly for 12 months by telephone and outpatient services (Table 4). 


\begin{tabular}{lcc} 
Table 4. Comparison of survival [N $(\%)]$. & & \\
\hline Group & & Survival \\
\cline { 2 - 3 } & 6 months & 12 months \\
\hline Research group & $28(93.3)$ & $25(83.3)$ \\
Control group & $30(90.9)$ & $20(60.6)$ \\
$P$ & $>0.05$ & $<0.05$ \\
\hline
\end{tabular}

\section{Changes in the $T$ cell subsets before and after treatment}

Compared with one week before treatment, the cluster of differentiation (CD) $3^{+}$, $\mathrm{CD}^{+}, \mathrm{CD}^{+} / \mathrm{CD}^{+}$in the study group were increased significantly 4 weeks after the treatment with statistical difference while the $\mathrm{CD}^{+}$in the control group with little increase showed no statistical difference (Table 5).

\begin{tabular}{l} 
Table 5. Before and after treatment changes in T cell subsets (\%). \\
\hline \begin{tabular}{lllllll}
\hline Group & $\mathrm{N}$ & \multicolumn{4}{c}{ T cell subsets } \\
\cline { 3 - 7 } & & \multicolumn{1}{c}{$\mathrm{CD}^{+}$} & $\mathrm{CD}^{+}$ & $\mathrm{CD}^{+}$ & $\mathrm{CD}^{+} / \mathrm{CD}^{+}$ \\
\hline Research group & One week before treatment & 30 & $58.28 \pm 6.08$ & $27.61 \pm 3.36$ & $29.48 \pm 7.14$ & $1.02 \pm 0.41$ \\
& 4 weeks after treatment & 30 & $68.15 \pm 5.10^{\Delta}$ & $35.60 \pm 5.29^{\Delta}$ & $29.19 \pm 7.20$ & $1.35 \pm 0.64^{\Delta}$ \\
Control group & One week before treatment & 33 & $58.19 \pm 6.12$ & $28.51 \pm 3.83$ & $27.91 \pm 5.05$ & $1.06 \pm 0.32$ \\
& 4 weeks after treatment & 33 & $58.06 \pm 5.74$ & $30.11 \pm 4.04$ & $26.58 \pm 4.92$ & $1.19 \pm 0.34$ \\
\hline
\end{tabular}
\end{tabular}

One week before treatment, ${ }^{\wedge} \mathrm{P}<0.05$.

\section{Adverse reactions in the two groups}

Adverse effects mainly included leukopenia, gastrointestinal reactions, radiation pneumonitis, and radioactive esophagitis. Adverse effect of degrees I and II were common. No significant difference was observed between the two groups. One case was excluded from the control group for the leukopenia of degree IV. In the study group, $5(16.7 \%)$ patients developed fever within 1-2 $\mathrm{h}$ after the reinfusion of DC-CIK and underwent spontaneous defervescence (Table 6).

Table 6. Comparison of adverse reactions [N $(\%)]$.
\begin{tabular}{lccccc}
\hline Group & $\mathrm{N}$ & Leukopenia & Gastrointestinal reactions & Radiation pneumonitis & Radioactive esophagitis \\
\hline Research group & 30 & & & & \\
I-II & & $23(76.7)$ & $21(70.7)$ & $10(33.3)$ & $8(26.7)$ \\
III & $2(6.7)$ & $0(0)$ & $3(10.0)$ & $1(3.3)$ \\
Control group & 33 & $25(75.8)$ & $24(72.7)$ & $12(36.4)$ & $10(30.3)$ \\
I-II & & $3(9.1)$ & $0(0)$ & $2(6.1)$ & $1(3.1)$ \\
III & $>0.05$ & $>0.05$ & $>0.05$ & $>0.05$ \\
\hline
\end{tabular}

\section{DISCUSSION}

According to the results of several large clinical trials, the 2003 American Society of Clinical Oncology Meeting proposed concurrent chemoradiotherapy as the standard regimen 
for the treatment of stage IIIB non-small cell lung cancer. Cisplatin acts as a non-specific agent on the cell cycle; it mainly reacts with the purine and pyrimidine bases of DNA, inhibits the replication of DNA, and destroys the structure of the cell membrane. Platinum-based drugs can increase major histocompatibility antigen-1 (MHC-I), and enhance the expression of tumor antigens in order to increase the recognition ability of DC and CIK cells to target tumor cells. Docetaxel acts as a specific agent on the cell cycle; it stabilizes the microtubules and resists depolymerization, blocking the tumor cells in the G2/M phase. Tumor cells in the G2/M phase are sensitive to radiotherapy, which enhances the curative effect during synchronous radiotherapy. Taxanes can improve the tumor lethality of $\mathrm{T}$ cells and NK cells. Concurrent chemotherapy can induce the tumor cells to release tumor antigens while killing them, in turn sensitizing them to DC and CIK cell therapy. The DC cell is one of the most powerful immune cells, and is a type of antigen presenting cell in the human body (Mehrian et al., 2013). Mature DC cells discharge chemokine DC-CK1 and the chemotaxis of naive T cells causes T cells to accumulate. They also show high expression of MHC-I and MHC-II molecules, B7-1, B7-2, CD86, CD80, CD44, CD40 and other stimulating factors and cell adhesion molecules including the intercellular adhesion molecule-1 (ICAM-1) and ICAM-3, which are beneficial for antigen presenting, T cell activation and CIK cell maturation. They secrete IFN- $\gamma$ and IL-12, induct T cells and NK cells to produce large amounts of TNF, perforin and granzyme, destroy the cell membrane structure of the target cell, activate cytosolic proteases, and finally lead to apoptosis of the tumor cell; CIK cells have a similar tumor-killing effect with NK cells, without the restriction of MHCs. They also have a strong anti-tumor activity like T lymphocyte (Jiang et al., 2006, 2013). Under the influence of chemokines, CIK cells can migrate to the tumor site, release perforin, and cause the cleavage of cytotoxic granules, resulting in lysis of the cancer cell. CIK cells showed high expression of the type II transmembrane glycoprotein, Fas ligand (FasL), which can bind to the type I transmembrane glycoprotein (Fas) (expressed by tumor cells) leading to the activation of the tumor cell-derived suicide program to cause tumor cell death. Currently DC, CIK, and other immune cells have multiple means of infusion, including intravenous, subcutaneous, intradermal, tumor bed, chest cavity, and lymphatic injections. In clinical practice, the most common way of delivering DC-CIK cells is by intravenous infusion; after intravenous transfusion, their in vivo distribution is beneficial for the treatment of lung cancer. In order to observe the differences in the distribution pattern for DC cells, Nicol et al. (2012) reinfused DC cells using different routes; they observed that the intravenous group could quickly disseminate to the lungs, liver, and spleen. The intradermal injection group could migrate to the lymph nodes, and the subcutaneous injection groups showed minor migration to the lymph nodes. The studies by Hazelrigg et al. (2002) have shown that after an intravenous injection, CIK cells rapidly dispersed within the body, first reaching the lung; after 2-6 h, the concentration of CIK peaked, then gradually decreased, and stabilized after 24 $\mathrm{h}$. The DC-CIK cells first reached the lungs at a high concentration, and stabilized for a long time, being beneficial in killing cancer cells.

The findings from the present study show that the RR in the study group was higher than that in the control group [83.3\% (25/30) vs 54.5\% (18/33)]. After treatment, the KPS in the study group was significantly higher than that in the control group. The 12-month survival rate in the study group was higher than that in the control group [83.3\% (25/30) to $60.6 \%(20 / 33)$, respectively], with statistical differences. However, there was no significant difference between the two groups in DCR, 6-month survival rate and adverse reactions. After treatment, the level of CEA, CA125, and CYFRA211 decreased significantly in the two 
groups, with statistical difference; however, there was no significant difference between the two groups. The $\mathrm{CD}^{+}, \mathrm{CD}^{+}, \mathrm{CD}^{+} / \mathrm{CD}^{+}$levels in the study group increased significantly at 4 weeks after receiving treatment as compared with the levels from one week before initiating treatment, with statistical differences, while the $\mathrm{CD}^{+}$in the control group showed no statistical difference, with a minor increase in levels. In summary, combined treatment with DC-CIK and concurrent radiotherapy and chemotherapy for the treatment of stage IIIB non-small cell lung cancer is better than radiotherapy and chemotherapy alone, and can improve quality of life, enhance immunity, and prolong survival.

\section{REFERENCES}

Bearz A, Minatel E, Abu Rumeileh I, Borsatti E, et al. (2013). Concurrent chemoradiotherapy with tomotherapy in locally advanced non-small cell lung cancer: a phase I, docetaxel dose-escalation study, with hypofractionated radiation regimen. BMC Cancer 13: 513 .

Casal Rubio J, Fírvida-Pérez JL, Lázaro-Quintela M, Barón-Duarte FJ, et al. (2014). A phase II trial of erlotinib as maintenance treatment after concurrent chemoradiotherapy in stage III non-small-cell lung cancer (NSCLC): a Galician Lung Cancer Group (GGCP) study. Cancer Chemother. Pharmacol. 73: 451-457.

Chang A (2011). Chemotherapy, chemoresistance and the changing treatment landscape for NSCLC. Lung Cancer 71: 3-10.

Hazelrigg MR, Hirsch JI and Merchant RE (2002). Distribution of adoptively transferred, tumor-sensitized lymphocytes in the glioma-bearing rat. J. Neurooncol. 60: 143-150.

Jiang J, Xu N, Wu C, Deng H, et al. (2006). Treatment of advanced gastric cancer by chemotherapy combined with autologous cytokine-induced killer cells. Anticancer Res. 26: 2237-2242.

Jiang J, Wu C and Lu B (2013). Cytokine-induced killer cells promote antitumor immunity. J. Transl. Med. 11: 83.

Mehrian M, Asemani D, Arabameri A, Pourgholaminejad A, et al. (2013). Modeling of tumor growth in dendritic cellbased immunotherapy using artificial neural networks. Comput. Biol. Chem. 29: 21-28.

Nicol AJ, Tazbirkova A, MacFarlane D, Nieda M, et al. (2012). Effect of cryopreservation, type of antigen and route of administration on in vivo migration of human monocyte-derived dendritic cells. J. Clin. Oncol. 30: el3128.

NSCLC Meta-Analyses Collaborative Group (2008). Chemotherapy in addition to supportive care improves survival in advanced non-small-cell lung cancer: a systematic review and meta-analysis of individual patient data from 16 randomized controlled trials. J. Clin. Oncol. 26: 4617-4625.

Pereira JR, Cheng R, Orlando M, Kim JH, et al. (2013). Elderly subset analysis of randomized phase III study comparing pemetrexed plus carboplatin with docetaxel plus carboplatin as first-line treatment for patients with locally advanced or metastatic non-small cell lung cancer. Drugs R D 13: 289-296.

Takayama K, Inoue K, Tokunaga S, Matsumoto T, et al. (2013). Phase II study of concurrent thoracic radiotherapy in combination with weekly paclitaxel plus carboplatin in locally advanced non-small cell lung cancer: LOGIK0401. Cancer Chemother. Pharmacol. 72: 1353-1359.

Tsuchida Y and Therasse P (2001). Response evaluation criteria in solid tumors (RECIST): new guidelines. Med. Pediatr. Oncol. 37: 1-3.

Yang LL, Ren BZ, Li H, Yu J, et al. (2013). Enhanced antitumor effects of DC-activated CIKs to chemotherapy treatment in a single cohort of advanced non-small-cell lung cancer patients. Cancer Immunol. Immunother. 62: 65-73.

Zhi X, Gao W, Han B, Yang Y, et al. (2013). VATS lobectomy facilitates the delivery of adjuvant docetaxel-carboplatin chemotherapy in patients with non-small cell lung cancer. J. Thorac. Dis. 5: 578-584. 